\title{
Comparison of Homocysteine and Heat Shock Protein 72 Responses Following Two Different Exercise Methods in Sedentary Women
}

\section{ARTICLE INFO}

\section{Article Type}

Original Research

\section{Authors}

Habibian M.*PhD

Monavari M. ${ }^{1} M S C$

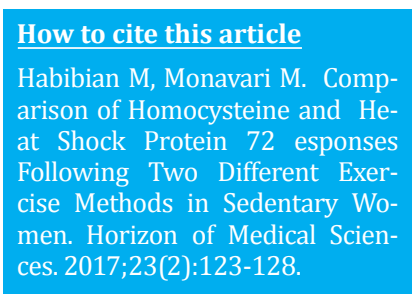

*Sports Sciences Department, Humanities Faculty, Qaemshahar Branch, Islamic Azad University, Qaemshahar, Iran

${ }^{1}$ Sports Sciences Department, Sari Branch, Islamic Azad University, Sari, Iran

\section{Correspondence}

Address: Humanities Faculty, Islamic Azad University, Allameh Tabarsi Street, Qaemshahar, Iran. Postal Code: 475161964 Phone: $+98(11) 42155025$ Fax: +98 (11) 42155117 habibian_m@yahoo.com

\section{Article History}

Received: May 2, 2016

Accepted: January 16, 2017

ePublished: March 25, 2017

\section{A B S T RA C T}

Aims Severe exercises lead to higher blood homocysteine levels, as well as heat shock protein 72 (HSP72). The aim of the study was to compare homocysteine and HSP72 responses following moderate-intensity continuous and high-intensity interval exercises in the sedentary women.

Materials \& Methods In the semi-experimental study, 20 sedentary and healthy young female students were studied in Islamic Azad University, Sari Branch, in 2014. The subjects, selected via purposeful and available sampling method, were randomly divided into two groups including continuous exercise and high-intensity interval exercise $(\mathrm{n}=10$ persons per group). 40-minute running on the treadmill with $60-65 \%$ of maximum consumed oxygen was conducted in continuous group. In addition, 36-minute running on the treadmill was conducted in interval group through alternate sets including 1 minute with $90-95 \%$ of maximum consumed oxygen and 3 minutes with $50 \%$ of maximum consumed oxygen. Blood sampling was done following 12-hour night fasting at 3 stages including before, immediately after, and 60 minutes after the exercises. Data was analyzed by SPSS 20 software using repeated ANOVA, LSD post-hoc, and independent T tests.

Findings Both severe exercises significantly increased homocysteine and HP72 levels $(\mathrm{p}<0.05)$, decreasing after 1-hour recovery compared to the interval immediately after the exercise. Nevertheless, HSP72 levels remained significantly higher than the basic levels $(\mathrm{p}<0.05)$.

Conclusion Both moderate-intensity continuous and high-intensity interval exercises similarly change homocysteine and HSP72 levels in the sedentary women.

\section{Keywords Exercise; Heat Shock Protein 72; Homocysteine}

\section{CITAT I O N L INKS}

[1] Plasma vitamins, amino acids, and renal function ... [2] Sport-related hyperhomocysteinaemia ... [3] Effect of short-term maximal exercise ... [4] Lipid profile and levels of homocysteine ... [5] Exercise, nutrition, and ... [6] Homocysteine status in former top-level ... [7] Cardiorespiratory fitness and plasma homocysteine ... [8] Homocysteine levels in children ... [9] Attenuation of homocysteine-induced ... [10] Exercise increases serum Hsp72 in ... [11] Different levels of Hsp72 in female ... [12] Exercise induces hepatosplanchnic ... [13] Exercise Intensity and Duration ... [14] The acute phase response and exercise ... [15] Physical activity and public health ... [16] Aerobic high intensity intervals improve V02max more than ... [17] Acute effects of three different circuit weight ... [18] High-intensity aerobic interval exercise in chronic ... [19] Generalized equations for predicting body density ... [20] High-intensity intermittent exercise attenuates ... [21] Total homocysteine in plasma or serum ... [22] Transient increase in homocysteine but not hyperhomocysteinemia ... [23] The impact of Wingate and progressive tests on homocysteine ... [24] Influences of acute resistance and aerobic exercises ... [25] Influences of acute and chronic aerobic exercise on the ... [26] Physical training decreases total plasma homocysteine and cysteine in ... [27] The Effects of Acute Exercise and Exercise Training ... [28] Heat shock proteins: modifying factors in physiological stress responses ... [29] Interaction of hsp 70 with newly synthesized ... [30] HSP70 peptidembearing and peptide- negative preparations ... [31] HSP expression in human leukocytes ... [32] Plasma cytokine changes in relation to exercise ... [33] The effect of aerobic and anaerobic tests on the alternation ... [34] The effect of one bout endurance and acute ... [35] Exercise-induced heat shock protein (HSP70) response ... [36] Glucose ingestion attenuates the exercise-induced ... [37] HSP70 expression: does it a novel fatigue signalling factor from immune... 
در برخى از مطالعات نشان داده شد كه فاكتورهاى شيوه زندكى از

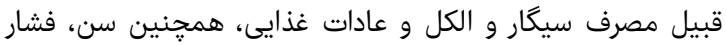

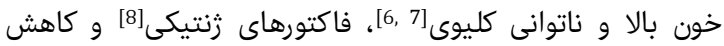

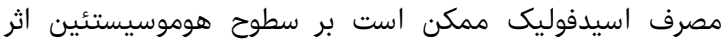

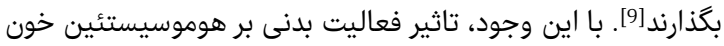

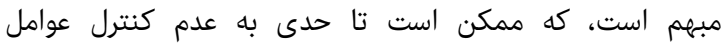

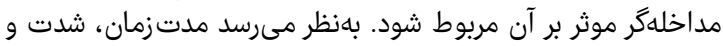

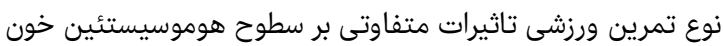
بكذارد و همجنين ممكن است سطوح آن وابسته به ميزان آمادگى تئى

جسمانى افراد باشد [5 فئ.

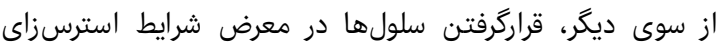

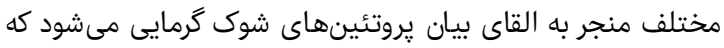

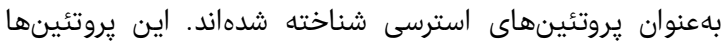

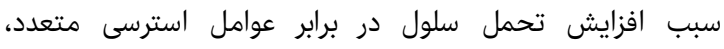

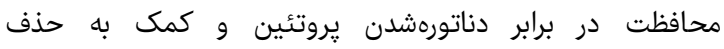

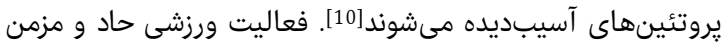

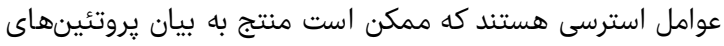

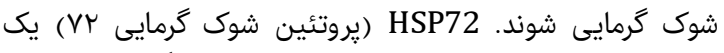

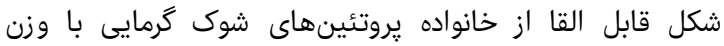

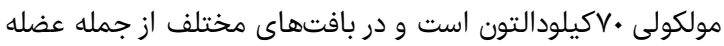

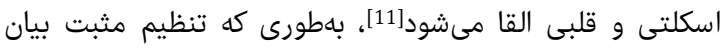

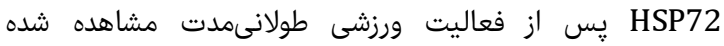

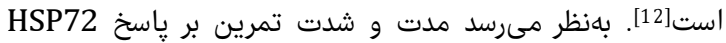

$$
\text { اثرگذار باشند[13]. }
$$

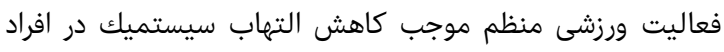

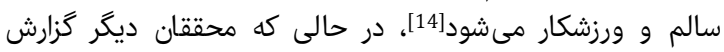

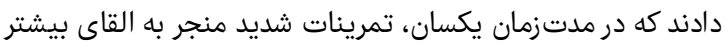

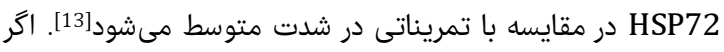

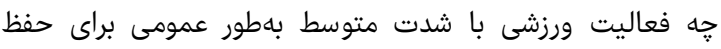

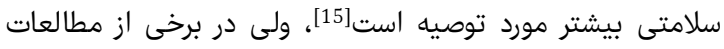

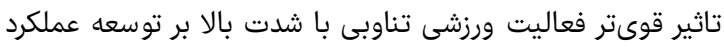

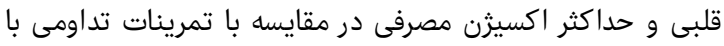

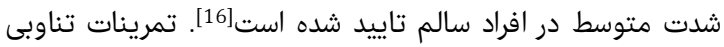

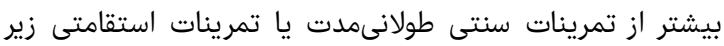

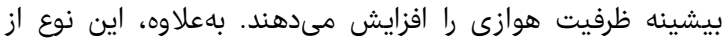

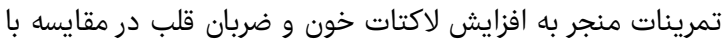

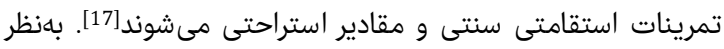

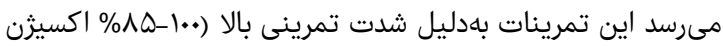

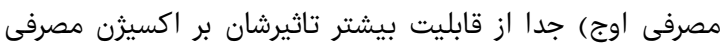

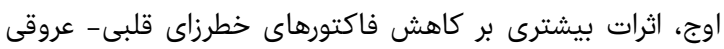

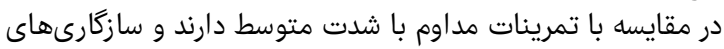

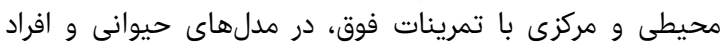

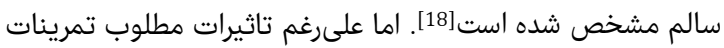

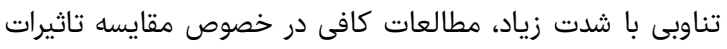
اين نوع تمرينات با تمرينات سنتى مداوم، بهويزات درات در زنان مشاهده

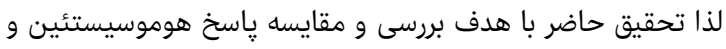

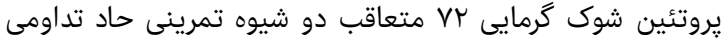
با شدت متوسط و تناوبى يرشدت دركات در زنان غيرفعال انجام شد.

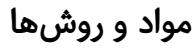

دوره سז، شماره r، بهار Tوس1

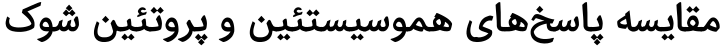

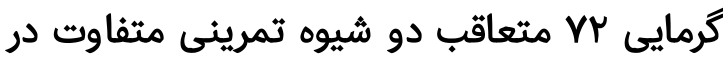 زنان غيرفعال}

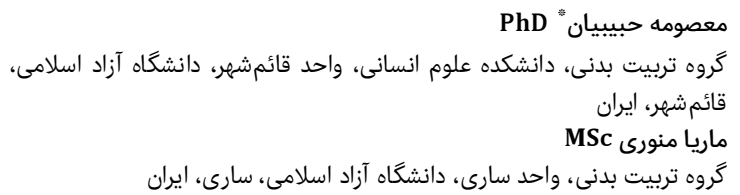

קكيده

اهداف: مطالعات نشان مىدهند فعاليت ورزشى حاد منجر به افزايش سطوح

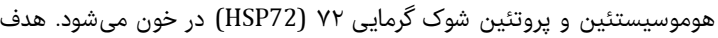

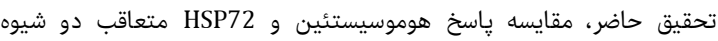

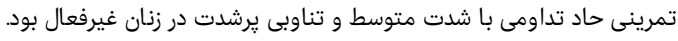

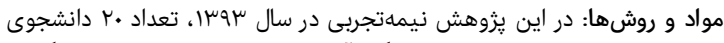

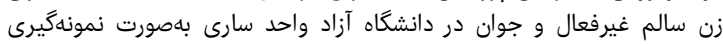

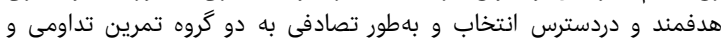

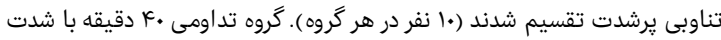

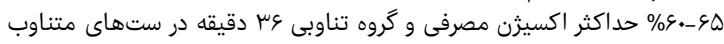

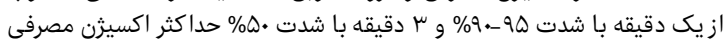

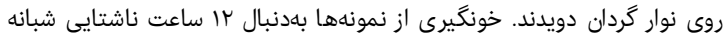

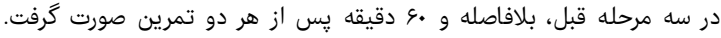

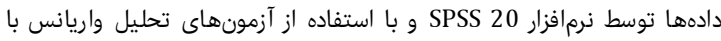

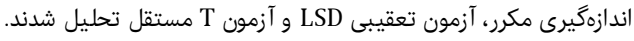

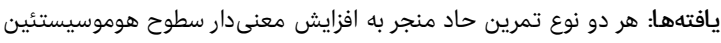

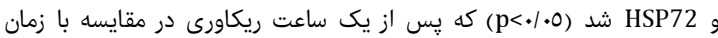

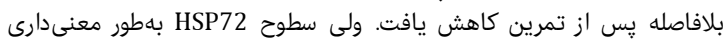

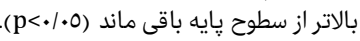

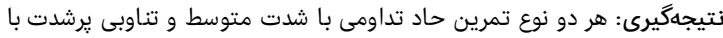

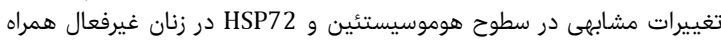

كليدوارهها: تمرين ورزشى، يروتئين شوك گرمايى Vr، هوموسيستئين

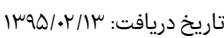

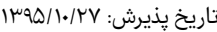
habibian_m@yahoo.com :نويسنده مسئول:

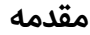

امروزه فعاليت جسمانى منظم بهاعنوان يك روش غيران ماردارويى

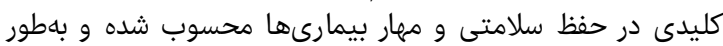

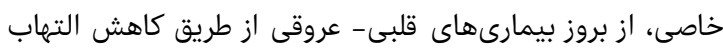

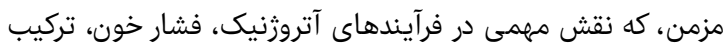

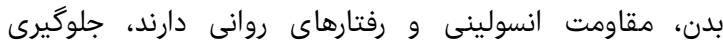

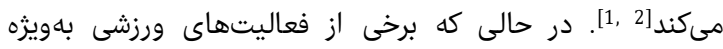

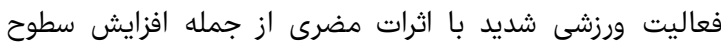

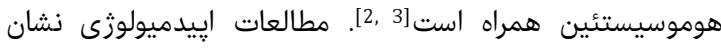

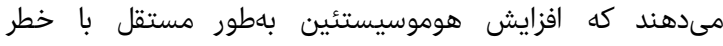

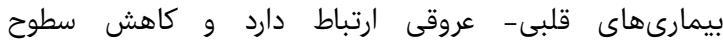

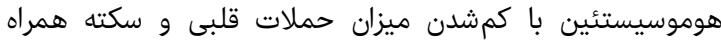

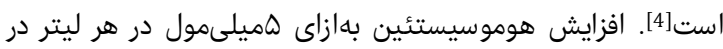

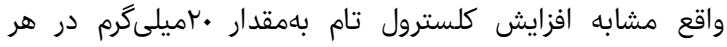

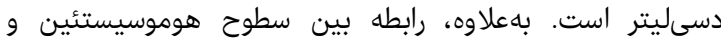
آترواسكلروز قوىتر از رابطه بين كلسترول و آترواسكلروز كزارش

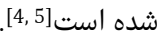

فصل نامه علمى - يروهشى افق دانش 


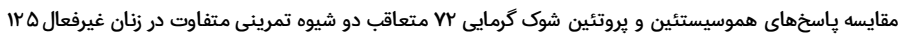

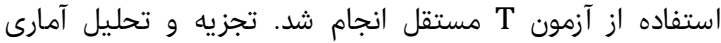
دادهها توسط نرم|فزار آس مSSS 20 صورت كرفت.

\section{يافتهها}

ميانكين مشخصات آنترويومترى آزمودنىهاى تحقيق در دو دو كروه

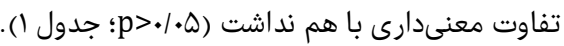

جدول () ميانكين آمارى شاخصهاى تركيب بدن آزمودنىها در كروههاى مورد

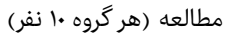

\begin{tabular}{|c|c|c|}
\hline سطح معنىدارى & كروه تمرين تداومى & كروه تمرين تناوبى \\
\hline ( & $r r / \Lambda \cdot \pm 1 / \varepsilon$. & 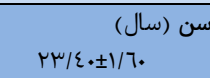 \\
\hline .1014 & $177 / 0 . \pm r / \mu$. & 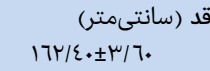 \\
\hline $.198 \mathrm{rr}$ & $O V / V \cdot \pm 0 / .$. & $\begin{array}{l}\text { وزن (كيلوكرم) } \\
O V / \Lambda \cdot \pm V / / .\end{array}$ \\
\hline . 1Y09 & 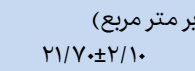 & 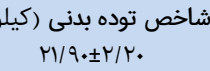 \\
\hline .1091 & $r 7 / q . \pm \mu / q$. & 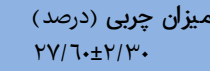 \\
\hline $.10 \mathrm{Vo}$ & \multicolumn{2}{|c|}{ 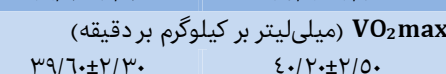 } \\
\hline
\end{tabular}

عامل زمان (قبل، بلافاصله و يك ساعت يس از تمرين) تاثير

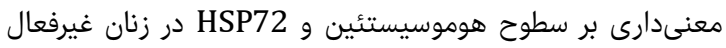

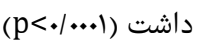

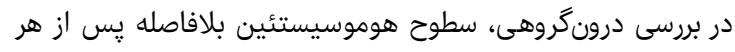

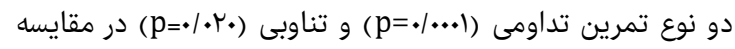

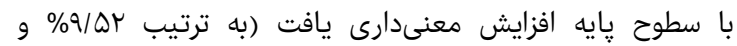

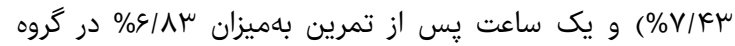

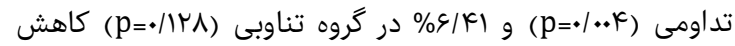
يافته و به سطوح پايه قبل از تمرين نزديك شد.

جدول r) مقايسه تغييرات سطوح هوموسيستئين و HSP72 در كروههاى تحقيق در مراحل مختلف هومسينين

\begin{tabular}{|c|c|c|}
\hline تمرين تناوبى & تمرين تداومى & شاخص \\
\hline & & هوموسيستئين (ميكرومول بر ليتر) \\
\hline$V / \varepsilon \cdot \pm \cdot / 09$ & V/RO & قبل از تمرين \\
\hline$V / ৭ 0 \pm \cdot / \varepsilon V$ & $1 / .0 \pm \cdot / 7 \varepsilon$ & بلافاصله يِ از تمرين \\
\hline$V / \varepsilon \varepsilon \pm \cdot / V$ & $\mathrm{~V} / 0 \cdot \pm \cdot / \mathrm{V}$ & يك ساعت يس از تمرين \\
\hline$\cdot 100 \pm \cdot / 1 \varepsilon^{* * *}$ & $\cdot / \mathrm{V} \cdot \pm \cdot / \cdot 7^{*}$ & اختلاف قبل با بلافاصله بعد \\
\hline$\cdot / \cdot \varepsilon \pm . / \cdot \Lambda$ & $\cdot / / \varepsilon \pm \cdot / \cdot V$ & اختلاف قبل با يك ساعت بعد \\
\hline$. / 01 \pm \cdot / 4$. & $.100 \pm .110^{.0}$ & اختلاف بلافاصله با يك ساعت بعد \\
\hline & & HSP72 (نانوگرم بر ميلىليتر) \\
\hline$\cdot / r 7 \pm * / \cdot \Lambda$ & $\cdot / r V \pm \cdot / \cdot V$ & قبل ازتمرين \\
\hline$\cdot / \mathrm{VY} \pm \cdot / 10$ & $\cdot / \wedge 0 \pm \cdot / 1 \varepsilon$ & بلافاصله يس از تمرين \\
\hline$. / \varepsilon r \pm \cdot / . q$ & $. / \varepsilon 7 \pm \cdot / .7$ & يك ساعت يس از تمرين \\
\hline$\cdot \mid \varepsilon 0 \pm \cdot / \cdot \mu^{*}$ & $\cdot 10 V_{ \pm} \cdot 1 \cdot \mu^{*}$ & اختلاف قبل با بلافاصله بعد \\
\hline$. / 10 \pm \cdot 1 \cdot r^{*}$ & $\cdot / 19 \pm \cdot / \cdot r^{*}$ & اختلاف قبل با يك ساعت بعد \\
\hline$\cdot / \cdot \varepsilon \pm \cdot / \cdot \varepsilon^{* * *}$ & $\cdot \mid \mu \wedge \pm \cdot / \cdot \varepsilon^{*}$ & اختلاف بلافاصله با يك ساعت بعد \\
\hline
\end{tabular}

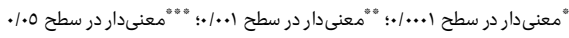

سطوح HSP72 بلافاصله يس از هر دو نوع تمرينات تداومى و

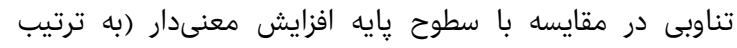

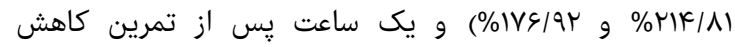
معنىدار (به ترتيب \% علىرغم اين كاهش، سطوح HSP72 در مقاديرى بيشتر از سطوح
اين يُوهش نيمهتجربى در سال سوسا در بين دانشجويان دختر آنا

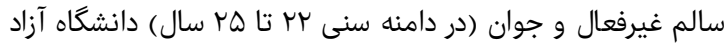

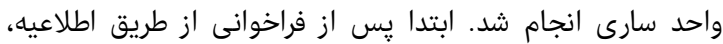

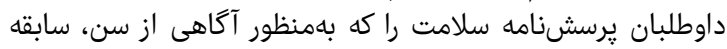

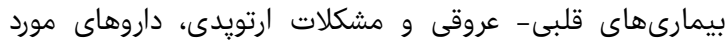

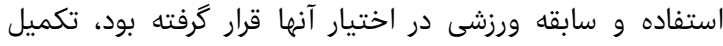

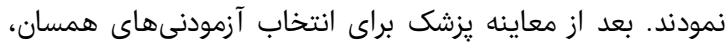

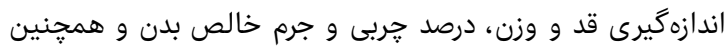

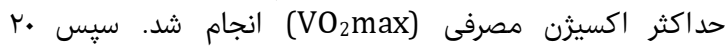

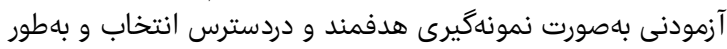

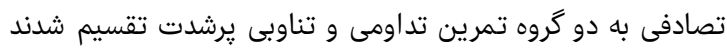

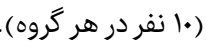

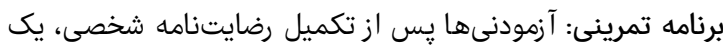

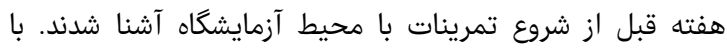

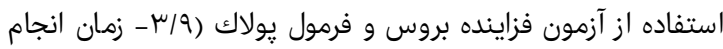

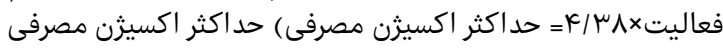

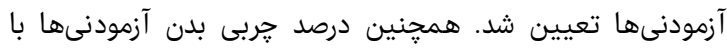

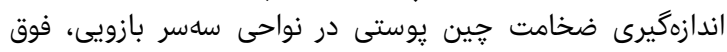

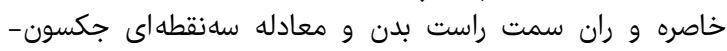

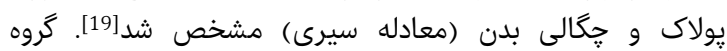

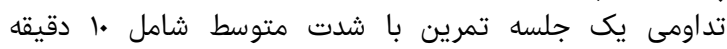

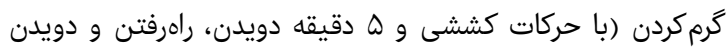

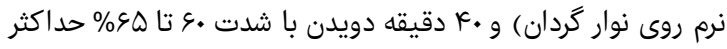

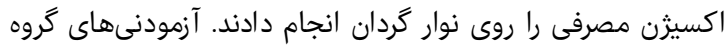

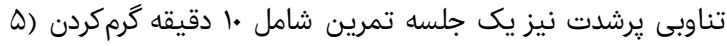

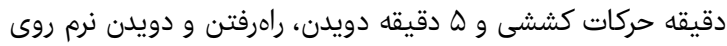

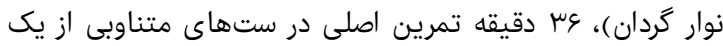

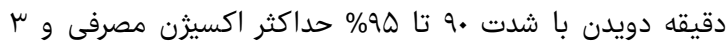

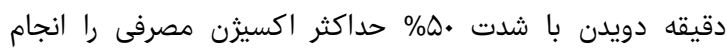

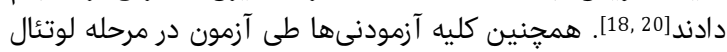

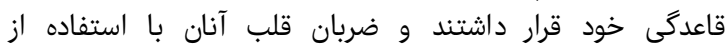

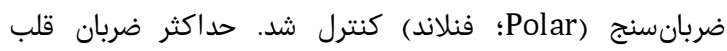

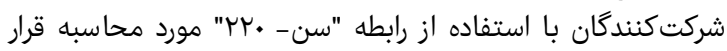
كرفت.

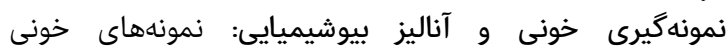

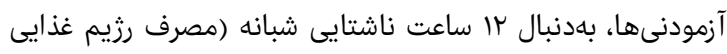

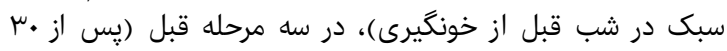

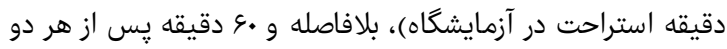

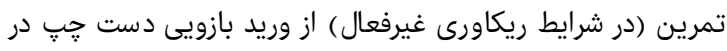

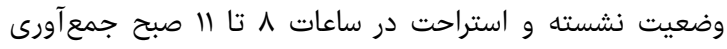

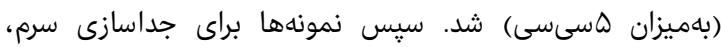

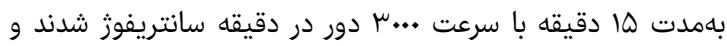

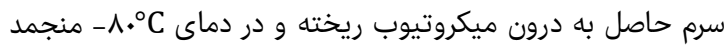

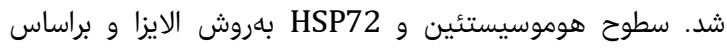

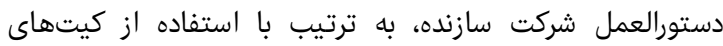

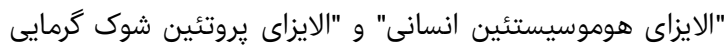

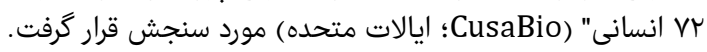

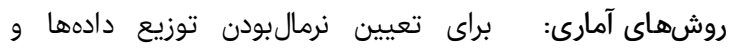

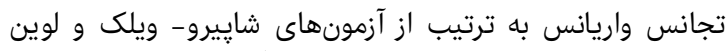

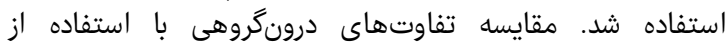

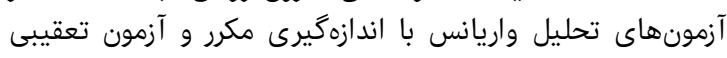

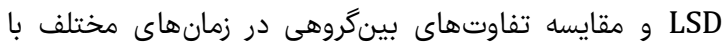


هموسيستين در افراد ورزشكار و غيرورزشكار همراه باشد و اين

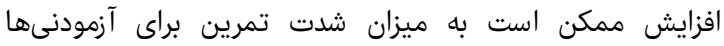

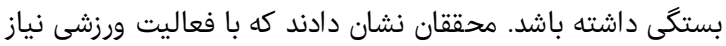

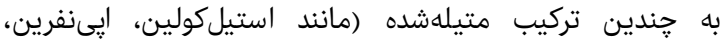

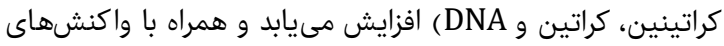

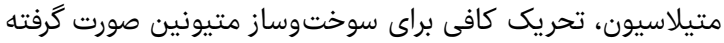

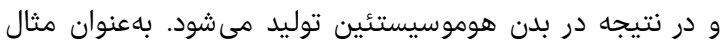

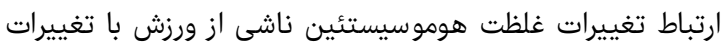

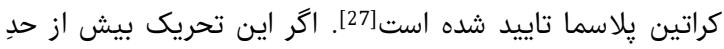

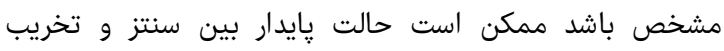

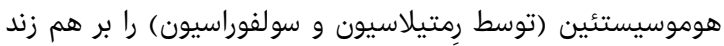

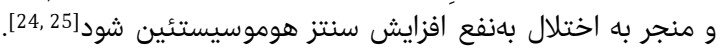

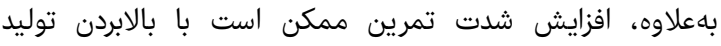

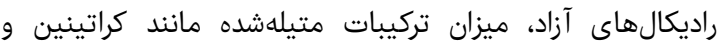

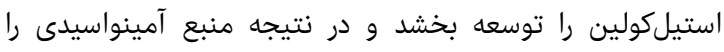

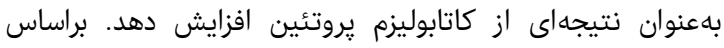

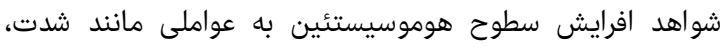

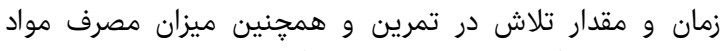

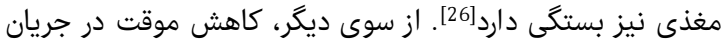

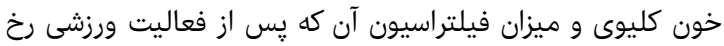

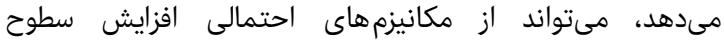

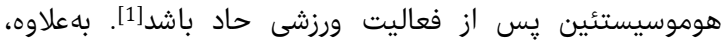

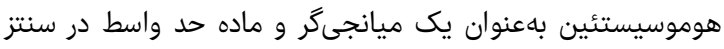

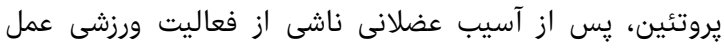

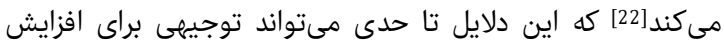

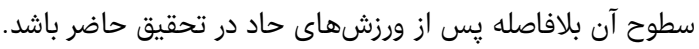

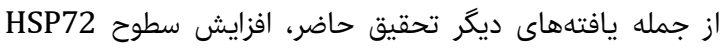

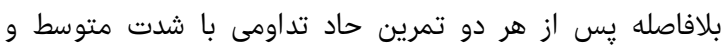

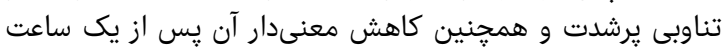

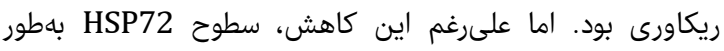

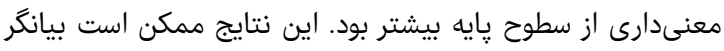

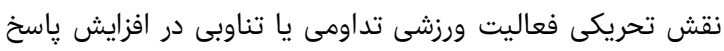

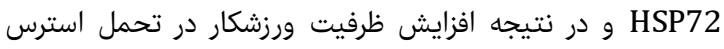

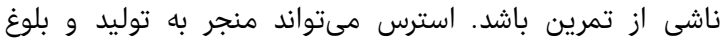

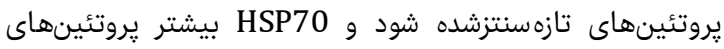

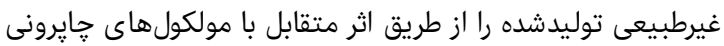

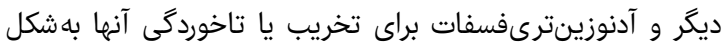

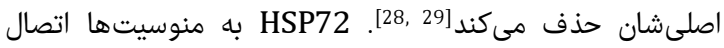

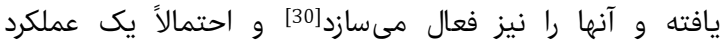

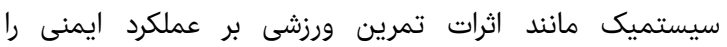

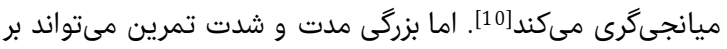

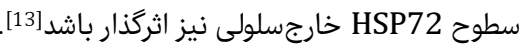

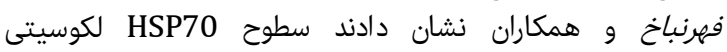

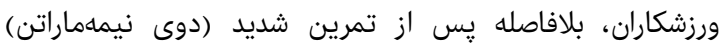

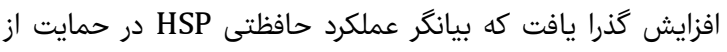

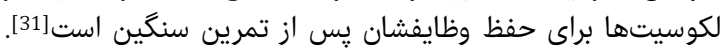

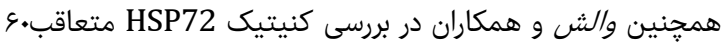

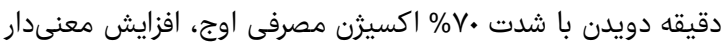

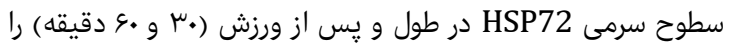

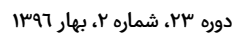

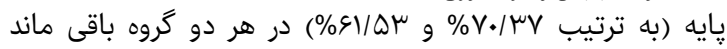

(p=./...1)

بين سطوح هوموسيستئين و HSP72 در گروههاى تداومى و

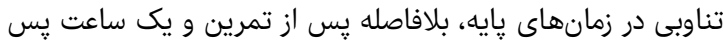

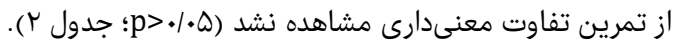

در تحقيق حاضر پاسخ هوموسيستئين و HSP72 متعاقب دو دوا

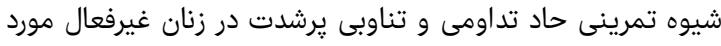

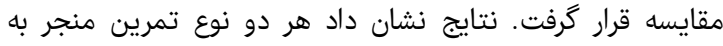

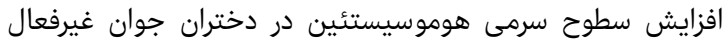

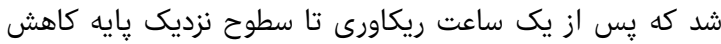

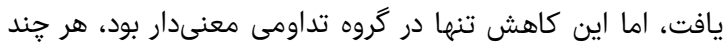

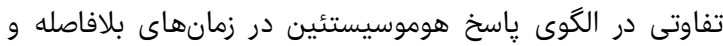

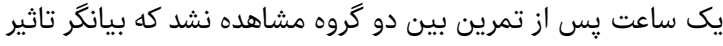

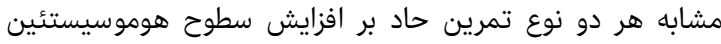

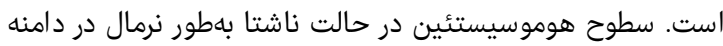

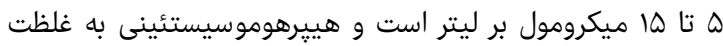

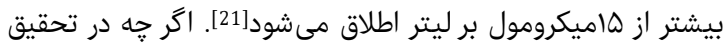

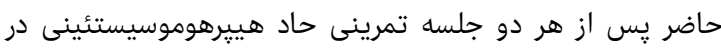

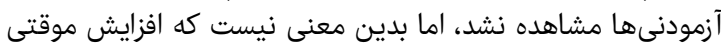

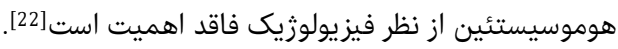

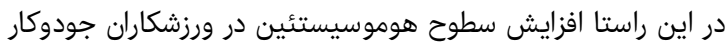

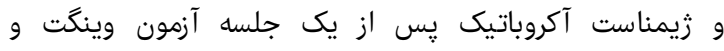

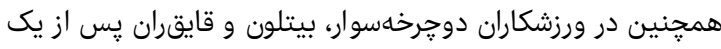

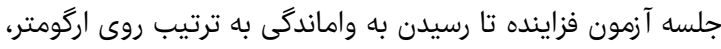

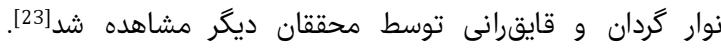

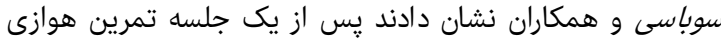

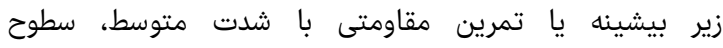

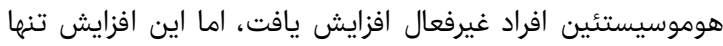

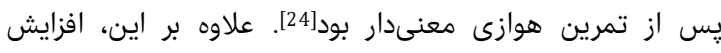

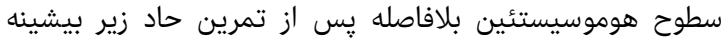

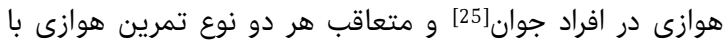

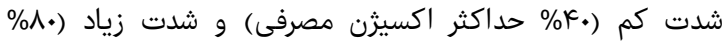

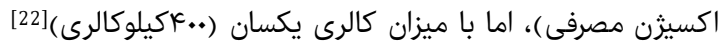

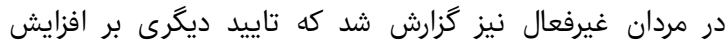

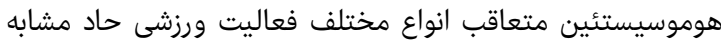

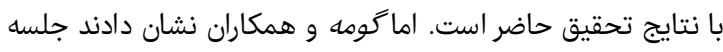

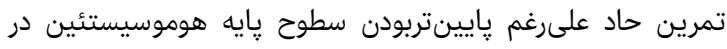

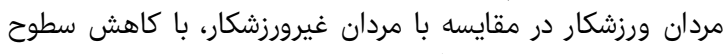

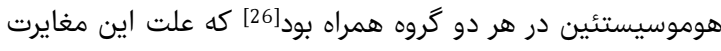

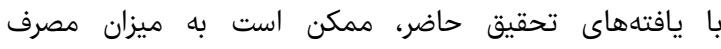

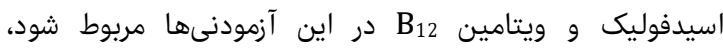

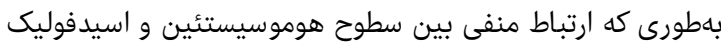

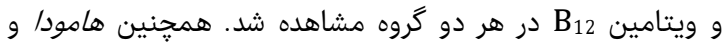

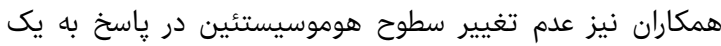

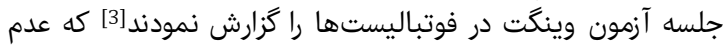

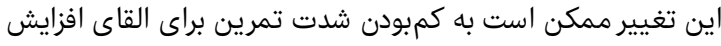

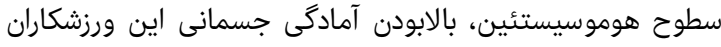

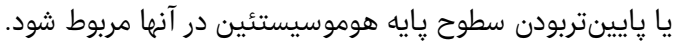

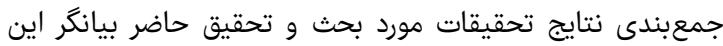

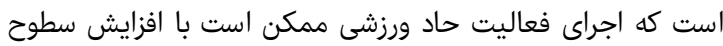




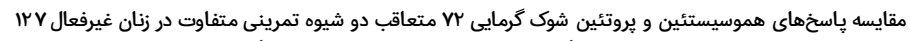

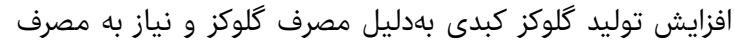

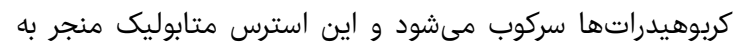

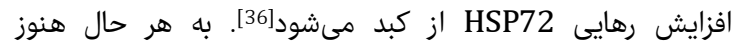

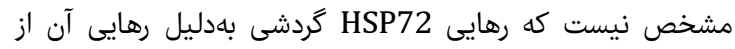

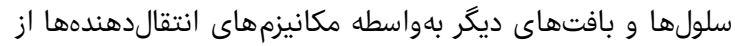

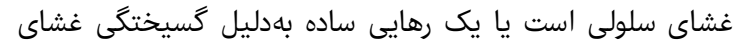

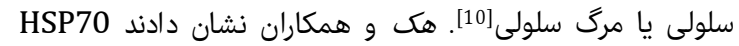

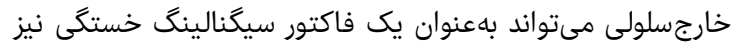

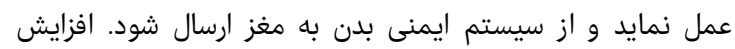

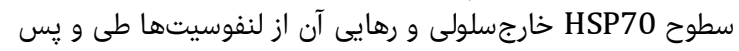

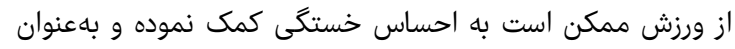

يك سيكنال خطر سيستم ايمنى بدن عمل إسن نمايد [37].

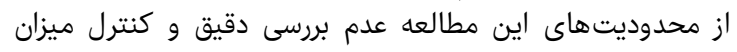

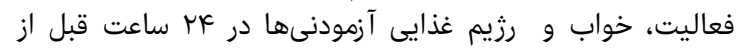

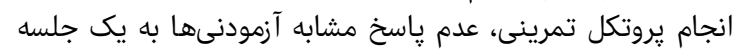

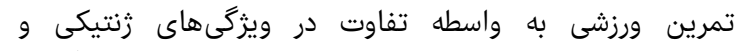

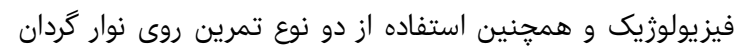

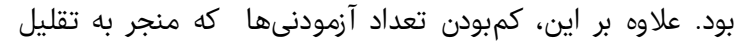

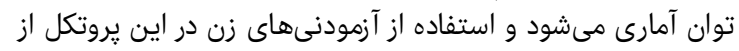

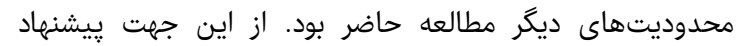

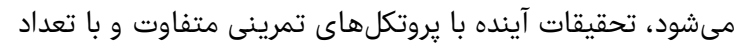
بيشترى از آزمودنىهاى مرد و زن، انجات إندام شود.

نتيجه

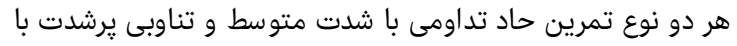

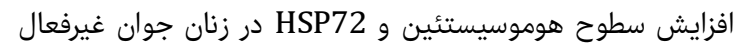

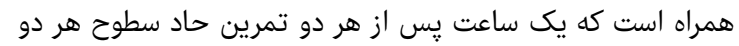

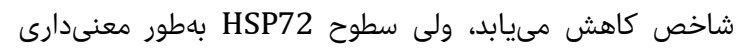
بالاتر از سطوح پايه باقى مئماند.

تشكر و قدردانى: بدين وسيله از كليه همكاران و دانشجويانى كه در

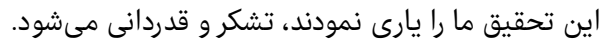

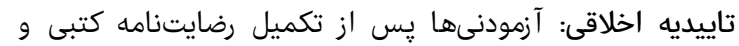

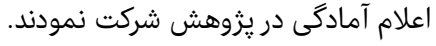

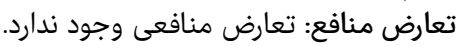

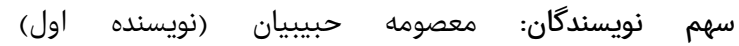

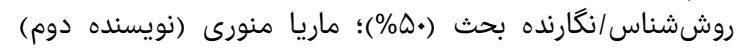

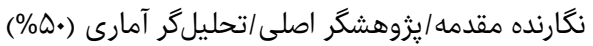

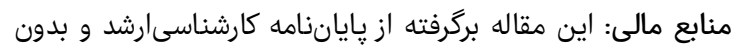
بهرهگيرى از منابع مالى سازمان و نهادى است.

منابع

1- Venta R, Cruz E, Valcárcel G, Terrados N. Plasma vitamins, amino acids, and renal function in postexercise hyperhomocysteinemia. Med Sci Sports Exerc. 2009;41(8):1645-51.

2- Borrione P, Rizzo M, Spaccamiglio A, Salvo RA, Dovio A, Termine A, et al. Sport-related hyperhomocysteinaemia: A putative marker of muscular demand to be noted for cardiovascular risk. Br J Sports Med. 2009;42(11):894-900.

3- Hammouda O, Chtourou H, Chaouachi A, Chahed H, Ferchichi S, Kallel C, et al. Effect of short-term maximal exercise on biochemical markers of muscle damage, total antioxidant status, and homocysteine levels in football
ززارش دادند. بهاعتقاد آنان، سطوح سرمى HSP72 ممكن است

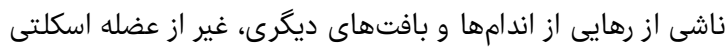

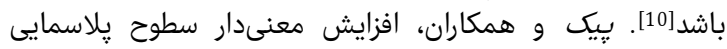

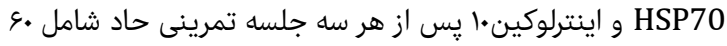

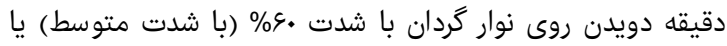

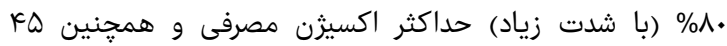

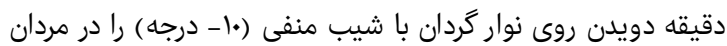

دونده مشاهده نمودند [32].

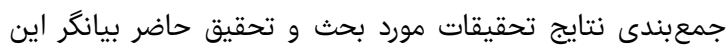

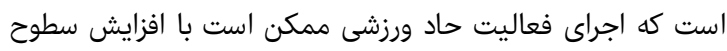

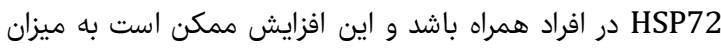

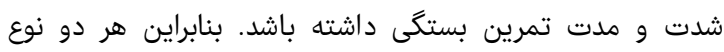

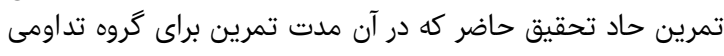

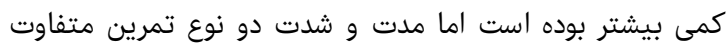

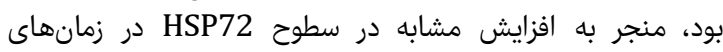

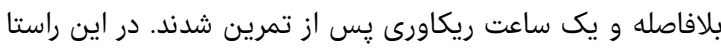

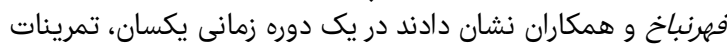
شديد منجر به القاى بيشتر

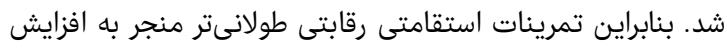

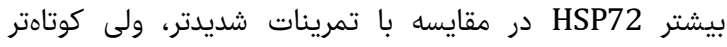

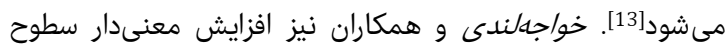

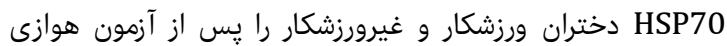

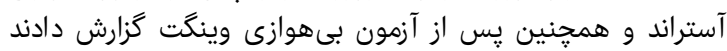

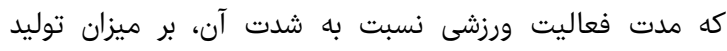

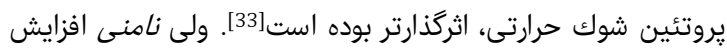

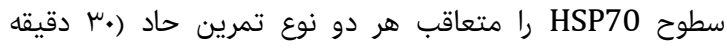

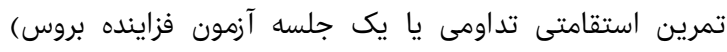

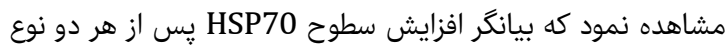

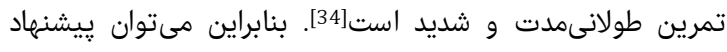

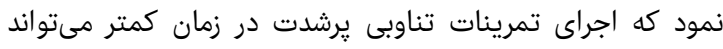

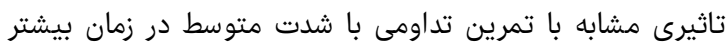

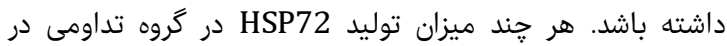

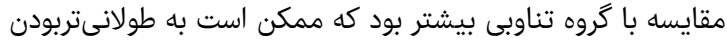

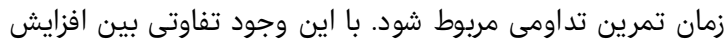

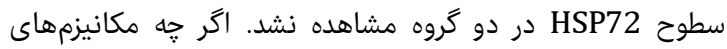

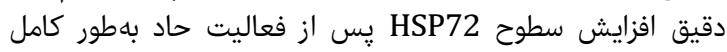

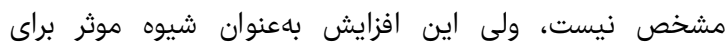

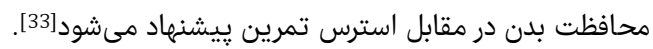

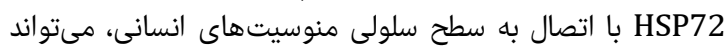

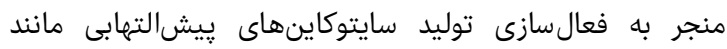

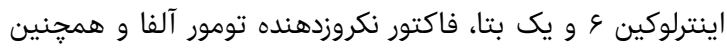

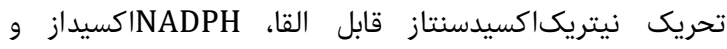

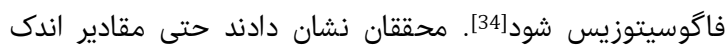
مى HSP72

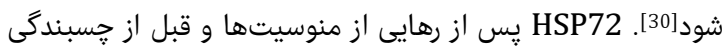

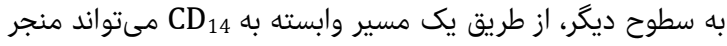

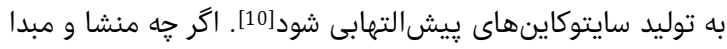

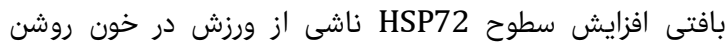
نيست، ولى مبدا اصلى رهايى

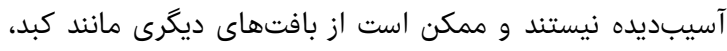

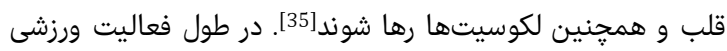


21- Ueland PM, Refsum H, Stabler SP, Malinow MR, Andersson A, Allen RH. Total homocysteine in plasma or serum: methods and clinical applications. Clin Chem. 1993;39(9):1764-79.

22- Iglesias-Gutiérrez E, Egan B, Diaz-Martínez AE, Penalvo JL, Gonzalez-Medina A, Martinez-Camblor P, et al. Transient increase in homocysteine but not hyperhomocysteinemia during acute exercise at different intensities in sedentary individuals. PLoS One. 2012;7(12):e51185.

23- Murawska-Ciałowicz E. The impact of Wingate and progressive tests on homocysteine, vitamin B6, B12 and folic acid levels in athletes' blood. Centr Eur J Sport Sci Med. 2014;8(4):5-17.

24- Subasi SS, Gelecek N, Ozdemir N, Orman M. Influences of acute resistance and aerobic exercises on plasma homocysteine level and lipid profiles. Turk J Biochem. 2009;34(1):9-14.

25- Gelecek N, Teoman N, Ozdirenc M, Pinar L, Akan P, Bediz C, et al. Influences of acute and chronic aerobic exercise on the plasma homocysteine level. Ann Nutr Metab. 2007;51(1):53-8.

26- Gaume V, Mougin F, Figard H, Simon-Rigaud ML, Nguyen UN, Callier J, et al. Physical training decreases total plasma homocysteine and cysteine in middle-aged subjects. Ann Nutr Metab. 2005;49(2):125-31.

27- Deminice R, Ribeiro DF, Frajacomo FT. The Effects of Acute Exercise and Exercise Training on Plasma Homocysteine: A Meta-Analysis. PLoS One. 2016;11(3):e0151653.

28- Kregel KC. Heat shock proteins: modifying factors in physiological stress responses and acquired thermotolerance. J Appl Physiol (1985). 2002;92(5):2177-86.

29- Beckmann RP, Mizzen LE, Welch WJ. Interaction of hsp 70 with newly synthesized: Implications for protein folding and assembly. Science. 1990;248(4957):850-4

30- Asea A, Kabingu E, Stevenson M A, Calderwood SK. HSP70 peptidembearing and peptide- negative preparations act as chaperokines. Cell Stress Chaperones. 2000;5(5):425-31.

31- Fehrenbach E, Passek F, Niess AM, Pohla H, Weinstock C, Dickhuth $\mathrm{HH}$, et al. HSP expression in human leukocytes is modulated by endurance exercise. Med Sci Sports Exerc. 2000;32(3):592-600.

32- Peake JM, Suzuki K, Hordern M, Wilson G, Nosaka K, Coombes JS. Plasma cytokine changes in relation to exercise intensity and muscle damage. Eur J Appl Physiol. 2005;95(5-6):514-21.

33- Khajehlandi A, Jafarei H, Mohammadi Demieh A, Barzideh P. The effect of aerobic and anaerobic tests on the alternation of heat shock proteins levels on young female. Arak Med Univ J. 2013;16(5):19-27. [Persian]

34- Nameni $F$. The effect of one bout endurance and acute exercise on HSP 70 in female. World Appl Sci J. 2012;18(5):727-30.

35- Sadowska-Krepa E, Klapcinska B. Exercise-induced heat shock protein (HSP70) response in human skeletal muscle and leukocytes. Med Sportiva. 2006;10(2):36-41. 36- Febbraio MA, Mesa JL, Chung J, Steensberg A, Keller $C$, Nielsen HB, et al. Glucose ingestion attenuates the exercise-induced increase in circulating heat shock protein 72 and heat shock protein 60 in humans. Cell Stress Chaperones. 2004;9(4):390-6.

37- Heck TG, Scholer CM, de Bittencourt PI. HSP70 expression: does it a novel fatigue signalling factor from immune system to the brain?. Cell Biochem Funct. 2011;29(3):215-26. players. Asian J Sports Med. 2012;3(4):239-46.

4- Sutken E, Akalin A, Ozdemir F, Colak O. Lipid profile and levels of homocysteine, leptin, fibrinogen and Creactive protein in hyperthyroid patients before and after treatment. Dicle Med J. 2010;37(1):1-7.

5- Joubert LM, Manore MM. Exercise, nutrition, and homocysteine. Int J Sport Nutr Exerc Metab. 2006;16(4):341-61.

6- Unt E, Zilmer K, Magi A, Kullisaar T, Kairane C, Zilmer M. Homocysteine status in former top-level male athletes: possible effect of physical activity and physical fitness. Scand J Med Sci Sports. 2008;18(3):360-6.

7- Dankner R, Geulayov G, Farber N, Novikov I, Segev S, Sela BA. Cardiorespiratory fitness and plasma homocysteine levels in adult males and females. Isr Med Assoc J. 2009;11(2):78-82.

8- Ruiz JR, Hurtig-Wennlöf A, Ortega FB, Patterson E, Nilsson TK, Castillo MJ, et al. Homocysteine levels in children and adolescents are associated with the methylenetetrahydrofolate Reductase $677 \mathrm{C}>\mathrm{T}$ genotype, but not with physical activity, fitness or fatness: The European youth heart study. Br J Nutr. 2007;97(2):255-62.

9- Hayward R, Ruangthai R, Karnilaw P, Chicco A, Strange $\mathrm{R}, \mathrm{McC}$ arty H, et al. Attenuation of homocysteine-induced endothelial dysfunction by exercise training. Pathophysiology. 2003;9(4):207-14.

10- Walsh RC, Koukoulas I, Garnham A, Moseley L, Hargreaves M, Febbraio MA. Exercise increases serum Hsp72 in humans. Cell Stress Chaperones. 2001;6(4):386-93.

11- Melo SF, Lunz W, Fontes EP, Dias CM, Carneiro MA Jr, Moura AG, et al. Different levels of Hsp72 in female rat myocardium in response to voluntary exercise and forced exercise. Arq Bras Cardiol. 2009;93(5):456-62.

12- Febbraio MA, Ott P, Nielsen HB, Steensberg A, Keller C, Krustrup P, et al. Exercise induces hepatosplanchnic release of heat shock protein 72 in humans. J Physiol. 2002; 544(Pt 3):957-62.

13- Fehrenbach EA, Niess $M$,Voelker K, Northoff $H$, Mooren FC. Exercise Intensity and Duration Affect Blood Soluble HSP72. Int J Sports Med. 2005;26(7):552-7.

14- Fallon K, Fallon S, Boston T. The acute phase response and exercise: court and field sports. $\mathrm{Br}$ J Sports Med. 2001;35(3):170-3

15- Haskell WL, Lee IM, Pate RR, Powell KE, Blair SN Franklin BA, et al. Physical activity and public health: Updated recommendation for adults from the American College of Sports Medicine and the American Heart Association. Circulation. 2007;116(9):1081-93.

16- Helgerud J, Hoydal K, Wang E, Karlsen T, Berg P, Bjerkaas $M$, et al. Aerobic high intensity intervals improve VO2max more than moderate training. Med Sci Sports Exerc. 2007;39(4):665-71.

17- Skidmore BL, Jones MT, Blegen M, MatthewS TD. Acute effects of three different circuit weight training protocols on blood lactate, heart rate, and rating of perceived exertion in recreationally active women. J Sports Sci Med. 2012;11(4):660-8.

18- Meyer P, Gayda M, Juneau M, Nigam A. High-intensity aerobic interval exercise in chronic heart failure. Curr Heart Fail Rep. 2013;10(2):130-8.

19- Jackson AS, Pollock ML, Ward A. Generalized equations for predicting body density of women. Med Sci Sports Exerc. 1980;12(3):175-81.

20- Sim AY, Wallman KE, Fairchild TJ, Guelfi KJ. Highintensity intermittent exercise attenuates ad-libitum energy intake. Int J Obes (Lond). 2014;38(3):417-22. 